\title{
On the Modeling of Patient-Specific Transcatheter Aortic Valve Replacement: A Fluid-Structure Interaction Approach
}

Giulia Luraghi , ${ }^{1}$ Francesco Migliavacca, ${ }^{1}$ Alberto Garci'A-González, ${ }^{2}$ Claudio Chiastra, ${ }^{1,3}$ Alexia Rossi, ${ }^{4}$ Davide CaO ${ }^{4}$ Giulio Stefanini, ${ }^{4}$ and Jose Felix Rodriguez Matas ${ }^{1}$

${ }^{1}$ Laboratory of Biological Structure Mechanics (LaBS), Department of Chemistry, Materials and Chemical Engineering

“Giulio Natta", Piazza L. da Vinci 32, 20133 Milan, Italy; ${ }^{2}$ Laboratori de Càlcul Numèric (LaCàN), E.T.S. de Ingenieros de Caminos, Canales y Puertos, Universitat Polite`cnica de Catalunya (UPC), Jordi Girona 1-3, 08034 Barcelona, Spain; ${ }^{3}$ PoliToBIOMed Lab, Department of Mechanical and Aerospace Engineering, Politecnico di Torino, Corso Duca degli Abruzzi 24, 10129 Turin, Italy; and ${ }^{4}$ Department of Biomedical Sciences, Humanitas University, via Rita Levi Montalcini 4, 20090 Pieve Emanuele, MI, Italy

(Received 14 March 2019; accepted 4 July 2019; published online 15 July 2019)

Associate Editor Ajit P. Yoganathan and Karyn Kunzelman oversaw the review of this article.

\begin{abstract}
Purpose-Transcatheter aortic valve replacement (TAVR) is a minimally invasive treatment for high-risk patients with aortic diseases. Despite its increasing use, many influential factors are still to be understood and require continuous investigation. The best numerical approach capable of reproducing both the valves mechanics and the hemodynamics is the fluid-structure interaction (FSI) modeling. The aim of this work is the development of a patient-specific FSI methodology able to model the implantation phase as well as the valve working conditions during cardiac cycles.

Methods-The patient-specific domain, which included the aortic root, native valve and calcifications, was reconstructed from CT images, while the CAD model of the device, metallic frame and pericardium, was drawn from literature data. Ventricular and aortic pressure waveforms, derived from the patient's data, were used as boundary conditions. The proposed method was applied to two real clinical cases, which presented different outcomes in terms of paravalvular leakage (PVL), the main complication after TAVR.

Results - The results confirmed the clinical prognosis of mild and moderate PVL with coherent values of regurgitant volume and effective regurgitant orifice area. Moreover, the final release configuration of the device and the velocity field were compared with postoperative CT scans and Doppler traces showing a good qualitative and quantitative matching.

Conclusion-In conclusion, the development of realistic and accurate FSI patient-specific models can be used as a support for clinical decisions before the implantation.
\end{abstract}

Keywords - Transcatheter aortic valve replacement (TAVR), Transcatheter aortic valve implantation (TAVI), Fluid-structure interaction simulation (FSI), Aortic valve, Patientspecific numerical model, Finite-element analysis (FE).

Address correspondence to Giulia Luraghi, Laboratory of Biological Structure Mechanics (LaBS), Department of Chemistry, Materials and Chemical Engineering "Giulio Natta", Piazza L. da Vinci 32, 20133 Milan, Italy.

Electronic mail: giulia.luraghi@polimi.it 


\section{Introduction}

Transcatheter aortic valve replacement (TAVR) is a minimally invasive procedure that is being increasingly adopted in the valvular diseases treatment (e.g. aortic stenosis) as it constitutes a solution for patients with adverse indications for standard surgery [1]. Recently, TAVR has been shown to represent a non-inferior alternative to classical surgical aortic-valve replacement (SAVR) not only for high-risk patients but also for intermediate-risk ones [2]. TAVR consists of the insertion of a stented valve in the aortic root using a catheter through the femoral, the subclavian or the carotid artery. In some cases, due to severe aortic stenosis, it is necessary to perform a balloon valvuloplasty to predilate the aortic annulus.

Nowadays, there are two families of transcatheter aortic valves approved by the Food and Drug Administration (FDA), namely balloon-expandable and self-expandable transcatheter aortic valves [3]. The former consists of a stent made by an elasto-plastic metal, the latter by a Nitinol super-elastic alloy. Both types of transcatheter aortic valves are equipped with tri-leaflet porcine or bovine pericardial tissue valve and an internal and/or external skirt to facilitate the leaflet anchoring to the stent and to reduce paravalvular leakage.

Despite transcatheter aortic valves are a proven technology since the first implantation in 2006 [4], many influential factors are still to be understood and require deeper investigation. In fact, TAVR procedure presents a different pattern of adverse events with respect to the SAVR [2]. From a hemodynamic viewpoint, the most common complication after TAVR is the presence of leaks [5], which undermines the long-term success of the implant [6]. In particular, paravalvular leaks (PVLs) appear due to the gap between the stent and the aortic wall, while supra-skirt leaks (SSL) happen when the prosthesis is placed too proximal with respect to the valvular annulus [7]. Post-procedural leaks are evaluated by means of echocardiography. PVLs are classified by the Valve Academic Research Consortium (VARC)-2 [8] in no leaks, mild (less than $30 \mathrm{~mL} /$ beat of regurgitant volume $(\mathrm{RV})$ and $0.10 \mathrm{~cm}^{2}$ of effective regurgitant orifice area (EROA)), moderate (30-59 mL/beat of RV and 0.10-0.29 $\mathrm{cm}^{2}$ of EROA) and severe (more than $60 \mathrm{~mL} /$ beat of RV and $0.30 \mathrm{~cm}^{2}$ of EROA) [5]. The post-procedural leaks have been evaluated in several experimental works for the most common commercially available devices [9-11]. Additionally, valve regurgitation can trigger other important complications after TAVR, such as hemolysis and thrombosis [12]. Recently, a high incidence of leaflets thrombosis following TAVR has been reported [13], in addition to dyspnea and increased gradients [14]. In this regard, the governing international standard for the development of transcatheter prosthetic heart valve (ISO 5840) requires the thrombus evaluation by using an integrated in-vitro, in-silico and ex-vivo approach. As a matter of fact, the FDA indicated the hemodynamics as key in the development of the leaflet thrombosis [15]. Leaflets thrombosis is also correlated with flow stagnation [9]; indeed, non-physiologic hemodynamic condition in Valsalva sinuses after transcatheter aortic valve implantation was detected with particle image velocimetry tests and was associated with stagnation zones at the base of the sinus in a reconstructed aortic root [16].

From a structural viewpoint, TAVR is associated with other complications. The transcatheter aortic valve position is a critical aspect because the device could migrate into the ventricle or in the aorta if it is deployed too low or too high with respect to the aortic annulus [17]. This issue, which is scarcely controlled during valve implantation, has obvious important consequences. Furthermore, the conduction system, situated in the interventricular septum, could be compromised after TAVR leading to an atrioventricular block with consequent permanent pacemaker requirement [18]. The eccentric distortion of the implanted device is strictly correlated with the dynamic leaflet deformations [19] and with the asymmetric opening 
of the leaflets, which could compromise the long-term valve durability [20]. Another complication is the coronary arteries occlusion, which could be caused by a misplacement of the device or by calcified native leaflets blocking the coronary inlets [21]. Finally, an important complication related to TAVR is the potential vascular damage provoked by the large-caliber sheaths used during the procedure, which can cause endothelial dysfunction and elevated circulatory levels of microparticles [22].

The previously mentioned complications are difficulty predictable by the clinicians because of the significant patient-to-patient variability in terms of geometry and morphology of the pathological valve. In this context, the development of tools able to reproduce the clinical procedure is crucial. Numerical simulations allow obtaining predictive information about the behavior of a medical device and understanding the interaction with the anatomical structures. The approach followed in the numerical studies on transcatheter aortic valves can be divided into idealized and patient-specific. The idealized approach aims at the investigation of numerical methodologies, technical aspects and specific clinical questions, with simplified models of complex structures [23,24,33,34,25-32]. The patient-specific approach is characterized by the segmentation and reconstruction of the anatomical geometry from computed tomography (CT) or magnetic resonance imaging [17,35,44-53,36,54-56,37-43]. This approach is more labor-intensive and time-consuming as compared to the first one but in favor of a more realistic representation of the clinical situation.

Numerical investigations include structural finite element (FE), computational fluid dynamics (CFD) and fluid-structure interaction (FSI) analyses. Table 1 concisely summarizes the main numerical studies performed on TAVR until now.

FE analyses allow the investigation of the structures to be performed, by solving the continuum mechanical equations. The crimping and release steps of the TAVR procedure were analyzed in terms of generated radial forces [24], by evaluating also the influence of the pericardium leaflets and skirt $[25,34,40]$. The presence of soft tissues in the numerical models involves the appropriate choice of the material constitutive law. This influences the resulted stress fields, which could be associated with clinical parameters, like the risk of inflammatory processes or annulus injuries [31]. In the literature, different material models for the aorta have been considered, from linear elastic to isotropic or anisotropic hyperelastic [39,55]. Moreover, the presence of calcification has a relevant impact on the TAVR outcome. In several studies $[30,37,42,55]$, calcium deposits and stenotic native valve were included in the numerical model of TAVR with various material models and approaches. In different FE works, the deployment of the transcatheter aortic valves in complete patient-specific models, including the aortic root, the native valve and the calcification, was simulated [40,44,47,49,52]. With similar complete models, TAVR positioning strategy and its consequences were investigated $[35,38,56]$. In fact, prosthesis positioning in TAVR procedure is a critical aspect; in particular, stent configuration, deformation and leaflets coaptation are affected by the device deployment zone, with affected post-operative valve performance. The best position, implantation depth and angle [17,36,46,51], size [50], and elliptical configuration [23] were analyzed in previous studies. CFD simulations give information on the pressure and velocity field within the fluid domain by solving the continuity and Navier-Stokes equations. PVL was numerically evaluated after FE deployment simulations with steady-state [45] or transient [53,57] CFD analyses. The variation of flow patterns in the aortic root-induced by transcatheter aortic valves [41] and the relation between valve thrombosis and reduced leaflets motion in case of TAVR [32] were 
investigated. In this latter, a CFD model with a moving boundary technique was implemented by exporting the valve kinematic from an FE analysis.

Finally, the FSI algorithm, which couples both FE and CFD analyses, represents the best numerical approach capable of reproducing the loading on the valve leaflets due to the fluid coupling [58]. In fact, the coupled solving of the structural kinematics and the blood fluid dynamics allows a physiological loading to be modeled. In the first FSI studies on TAVR $[28,43]$, only the coupling between blood and the aorta or blood and the valve was considered. On the one hand, the design of different transcatheter aortic valves was considered in the aorta geometry, neglecting the native valve leaflets and related calcifications [43]. On the other one, a comparison between structural FE and FSI approach on a generic tri-leaflets transcatheter aortic valves was performed, confirming the more realistic representations of the valve behavior when applying the FSI method [28]. TAVR was also compared to the conventional surgical valve replacement with FSI simulations in terms of leaflet stresses and thromboresistance profile [59].

To the best of our knowledge, there are only two FSI works on the complete TAVR procedure $[48,54]$. In both studies, the deployment of the complete device, frame, leaflets, and skirt was performed. However, the patient-specific domain was simplified by considering the aorta as a rigid part [54] or with a linear elastic material law [48]. In both cases, the native valve and calcification were neglected, and the boundary conditions applied to the fluid domain were not patient-specific, in particular idealized pressure curves [48] or from specific lumped-parameter models [54].

In this context, the aim of this work is the development of a robust framework to perform patient-specific FSI simulations of self-expandable transcatheter aortic valves. The proposed method was applied to two real clinical cases, which presented different outcomes, with mild and moderate PVL after TAVR. The purpose of this work is not to provide specific clinical indications for the patient-specific procedure, but to present a new and versatile numerical methodology to study the TAVR in its whole.

Furthermore, the patient-specific domain includes an anisotropic hyperelastic model for the aorta and the presence of the native valve and calcifications. In particular, in this work (i) a parametrical CAD model, similar to the real design of the implanted valve with the frame and the pericardium tissue, was created; (ii) the morphology of the native aortic root and calcifications was reconstructed from CT images of the patients, while native valves were manually drawn; (iii) all the components of the TAVR model were discretized and the material properties were assigned; (iv) two FSI simulations were carried out, by applying patientspecific boundary conditions; finally (v), the results of the simulations were compared with the available post-procedural clinical data.

\section{Methods}

\section{$\underline{\text { Patient data }}$}

Data on two patients (A and B) undergoing the same aortic bioprosthetic valve implantation but who experienced different procedural outcomes were obtained retrospectively. Both patients were symptomatic for severe aortic stenosis and considered eligible for TAVR through femoral access.

Prior medical history of patient A was relevant for ischemic cardiomyopathy for which he underwent surgical and percutaneous myocardial revascularization in the past. Conversely, patient B did not have any previous cardiovascular event. 
Preoperative assessment by means of transthoracic echocardiography confirmed the severity of the aortic stenosis with mild to moderate left ventricular systolic dysfunction in both patients. Mean aortic transvalvular gradient of patient A and patient B was $42 \mathrm{mmHg}$ and $46 \mathrm{mmHg}$ respectively. CT scans, regularly performed before TAVR, showed almost equivalent aortic valve annular measurements and an annular perimeter of $80 \mathrm{~mm}$ in both cases. Hence, the same aortic valve bioprosthesis, Medtronic CoreValve Evolute R size 29 (Medtronic Inc, Minneapolis, Minnesota), was selected for implantation in patient A and B.

The two TAVR procedures were successfully performed at the same institute (Humanitas Research Hospital, Milan, Italy), and no major complication occurred.

At the time of intervention, patient A underwent balloon predilation of the aortic stenosis followed by transcatheter valve implantation. Postprocedural angiography of patient A showed good bioprosthesis expansion and sealing with only mild PVL. At a heart rate of $70 \mathrm{bpm}$, invasive aortic and ventricular pressure in patient A was $123 / 50 \mathrm{mmHg}$ and $126 / 7 \mathrm{mmHg}$ respectively, meaning no residual transvalvular gradient. The patient was discharged home on life-long aspirin monotherapy.

Conversely, Patient B underwent direct transcatheter valve implantation without prior predilation of the stenotic valve. Postprocedural angiography showed acceptable although slightly low valve implantation across the aortic annulus and a moderate PVL due to a double regurgitation jet located anteriorly and posteriorly to the prosthesis. No evidence of significant antegrade transvalvular gradient was shown by aortic and ventricular pressure curves, being $160 / 55 \mathrm{mmHg}$ and $169 / 9 \mathrm{mmHg}$ respectively. Patient B agreed on participating in a clinical randomized study and was discharged home on an experimental dual antithrombotic therapy. Clinical follow-up at 2 years was available for both patients and none of them reported any significant adverse event, such as hospital readmission for cardiovascular cause or persistence of symptoms after intervention. Device migration was not observed in the two patients. Echocardiographic findings at 3 months for patient $\mathrm{A}$ and at 12 months of patient $\mathrm{B}$ remained overall unchanged compared to early postoperative assessment. The two patients were both included in a prospective registry, approved by the Institutional ethics committee.

\section{Prosthesis model}

A high-fidelity model of the CoreValve Evolute R (Medtronic, Minneapolis, MN, USA) was created using the illustrations in the literature [53]; in particular, a complete parametric CAD model of the frame, leaflets, and skirt was realized by means of SolidWorks 2018 (Dassault Systèmes SolidWorks Corp., Waltham, MA, USA). For the metallic frame, a constant-diameter stent was obtained by replicating the elementary unit cell 15-times (Figure 1a). A deform function was then applied to reach the final non-constant diameter shape, by following the profile of the device. The leaflets and the skirt were built in accordance with the final stent configuration (Figure 1b). Sewing suture between the frame and pericardial tissue and between leaflets and skirt were neglected [60]. The stent was discretized with 159,435 hexahedral linear elements (Figure 1c) with reduced integration formulation and Puso hourglass control [61]. After a sensitivity analysis, 3 elements in both the width and the thickness of the stent were found to be enough to capture the strain and stress fields. In fact, the difference in terms of first principal stress between meshes with three or four elements in selected elements during the crimping phase was less than $4 \%$. In accordance with the stent grid, the leaflets were meshed with 5,706 Belytschko-Lin-Tsay quadrilateral linear shell elements [62] with one-point integration and viscosity hourglass control [63]. In our previous work [64], we demonstrated 
the best performance of this formulation for shell heart valve models. 32,388 Belytschko-Tsay [65] triangular membrane elements with viscosity hourglass control were used to discretize the thin skirt (Figure 1c). The thickness of the pericardium tissue for both the leaflets and the skirt was of $0.4 \mathrm{~mm}$ [60]. The meshing procedure of all the prosthesis components was done by means of ANSA Pre Processor v19.0 (BETA CAE Systems, Switzerland).

The mechanical properties of pseudo-elastic NiTi were taken from the literature [46]. Due to the weak hyperelastic behavior of the porcine pericardium found in the literature [66], the leaflets and skirt were modeled with a linear elastic Young's modulus of $1 \mathrm{MPa}$, a Poisson's ratio of 0.45 , and density of $1100 \mathrm{~kg} / \mathrm{m}^{3}$ [54,67]. Moreover, for the working strain range, the material behavior is well represented with the initial tangent modulus of the hyperelastic characteristic curve.

The stent and the pericardium were fixed together with a node-to-node connection in the grids, although the sewing sutures were not modeled. A penalty self-contact was defined between the three leaflets.

\section{Patient-specific models}

To obtain 3D reconstructions of the aortic root and the calcium deposits, the pre-operative CT scans of the two patients were processed using the software Mimics (Materialise, Leuven, Belgium). In particular, the CT images corresponding to the end-diastolic phase of the cardiac cycle were segmented using grey-value thresholding followed by morphologic operations. The obtained segmented masks were then used to derive triangulated meshes representing the $3 \mathrm{D}$ models of the aortic root and the calcium deposits.

A quantitative comparison between the clinical measurements of the aortic annulus and the segmented one was performed. The estimated diameters of the segmented models, $26.5 \mathrm{~mm}$ in case A and $25.1 \mathrm{~mm}$ in case B, were within the clinical dimensions of $21 \mathrm{~mm}$ x $29 \mathrm{~mm}$. These results confirmed the correct choice of the device size $29 \mathrm{~mm}$, which is indicated for annulus size of 23-27 $\mathrm{mm}[68]$.

The surface lumen of the aorta was extruded in the radial direction to obtain a constant thickness of $2 \mathrm{~mm}$ [35]. The aorta was discretized into 35,640 hexahedral linear fully integrated solid elements with hybrid formulation to avoid pressure locking [69]. A hex-block method, developed in ANSA Pre Processor v19.0.x platform (BETA CAE Systems, Switzerland) permitted the generation of a fully mapped hexahedral grid. To draw the native valve, the leaflet surfaces were generated by following reference points identified at the commissures and basal leaflet attachment lines on the aortic lumen [39]. The native valve leaflets were then meshed with 1,859 Belytschko-Lin-Tsay quadrilateral linear shell elements [62] with one-point integration and viscosity hourglass control [63] (Figure 2c-2d). The thickness of the native valve was fixed as $0.5 \mathrm{~mm}$ [35]. The segmented calcium deposits, after smoothing and wrapping process, were discretized with 38,429 one-point nodal pressure tetrahedral elements (Figure 2c-d).

The aorta material was modeled using a user-defined material to describe an anisotropic hyperelastic constitutive law. The strain energy function consisted of a modified HolzapfelOgden function $[70,71]$ in which the isotropic term has been augmented:

$$
\Psi=D_{1}\left[\exp \left\{D_{2}\left(\overline{I_{1}}-3\right)\right\}-1\right]+\frac{k_{1}}{2 k_{2}}\left[\exp \left\{k_{2}\left(\overline{I_{4}}-1\right)^{2}\right\}-1\right]+\frac{\kappa}{2}(J-1)^{2}
$$


where $D_{1}$ and $\mathrm{k}_{1}$ are stress-like material constant, whereas $D_{2}$ and $\mathrm{k}_{2}$ are dimensionless material parameters, and $\kappa$ is the bulk modulus. $\bar{I}_{1}$ is the first invariant of the modified right CauchyGreen strain tensor, $\bar{I}_{1}=\operatorname{tr} \bar{C}$, and $\bar{I}_{4}$ is the pseudo-invariant of the right Cauchy-Green strain tensor, $\bar{I}_{4}=a_{0} \cdot \bar{C} a_{0}$, with $a_{0}$ a unit vector along the direction of anisotropy coincident with the circumferential direction of the artery. We adopted this formulation do to the lack of histologic evidence regarding the fiber distribution in aortic tissue. In addition, biaxial tests conducted on aorta [72] indicated the circumferential direction as the stiffer. The material parameters for the strain energy function in Eq. (1) were obtained by means of a nonlinear regression analysis of the mean biaxial test for aortic tissue reported in [72], resulting in $D_{1}=0.214 \mathrm{kPa}, D_{1}=41.3, k_{1}=0.212 \mathrm{kPa}, k_{2}=130$ and $\kappa=10^{4} \mathrm{kPa}[71]$.

To obtain a homogenous and smooth distribution of the fibers, a specific local coordinate system was defined for each finite element of the aorta, according to the two principal stress directions obtained with a pre-analysis [73,74] (Figure 2a). The two directions overall drew the circumferential and the axial directions [75] (Figure 2b).

The native valve and calcifications were modeled as linear elastic materials with a density of $1100 \mathrm{~kg} / \mathrm{m}^{3}$, Young's modulus of $4 \mathrm{MPa}$ and Poisson's ratio of 0.45 [7] for the valve; and a density of $2000 \mathrm{~kg} / \mathrm{m}^{3}$, Young's modulus of $12.6 \mathrm{MPa}$ and Poisson's ratio of 0.45 [76] for the calcifications. A node-to-surface tied contact was defined between the commissural edges of the native valve and the internal surface of the aorta, whereas a surface-to-surface tied contact was defined between the calcifications and the leaflets.

\section{FSI set-up simulation}

The finite element simulations were performed on computer node with 4 CPU Xeon E5-4627 v3 with 10 cores per CPU and 256 GB of RAM memory using the commercial finite element solver LS-Dyna 971 Release 10.1 (LSTC, Livermore CA, USA). A damping coefficient sensitivity analysis was performed for each step of the simulations for each structural part in order to smooth the frequency vibration without introducing numerical artificial viscosity [64]. A quasi-static condition in each step was achieved as the ratio between the kinetic and the internal energy was found to be less than $5 \%$ during all the simulated cardiac cycles [77].

A selective mass-scaling was adapted to keep the time-step at a constant $10^{-6} \mathrm{~s}$ during the simulations.

\section{1st step: insertion of the crimped device in the stenotic native valve}

To create the space to insert the catheter with the crimped device in the stenotic valve, a predilatation of the valve was carried out within a rigid catheter with a diameter of $10 \mathrm{~mm}$.

\section{2nd step: transcatheter aortic valves implantation}

The diameter of the valve was uniformly crimped down to $9 \mathrm{~mm}$ by twelve rigid planes (not shown in Figure 1) in $0.7 \mathrm{~s}$. The crimped diameter value was enough to allow the following positioning into the patient's domain; lower values lead to numerical instability of the crimped structures [54]. Once the device was crimped, it was released in a $10 \mathrm{~mm}$ diameter catheter, which was positioned coaxially to the aorta. The rigid catheter was slowly lifted from the annulus to the ascending aorta (Figure 1d). The prosthesis valve annulus was positioned on the same plane of the native one, following the indication of the interventional cardiologist. A penalty contact with friction coefficient of 0.1 [78] was defined between the device and the 
aorta, the native valve and the calcifications. As boundary conditions, the aorta was fixed at the two external ends in all directions.

\section{3rd step: two cardiac cycles after TAVR}

The "operator split" Lagrangian-Eulerian approach [79], a non-boundary fitted method implemented in the solver LS-Dyna, was adopted to define the interaction between the solid structure (valve) and the fluid (blood). In this approach, the structural forces calculated on the actual nodes are distributed to the fluid, whose equations are "split" into Lagrangian and advection steps.

A fluid domain was built in order to immerse all the structural parts of the model and meshed with the same element size of the aorta to prevent any leakage [64], with a total number of 113,216 hexahedral Eulerian one- point elements (Figure 2c-2d). An appropriate number of coupling nodes was set to prevent any numerical leakage through the structures.

The fluid domain consisted of a main volume, inlet, outlet, and external parts. The patientspecific ventricular and aortic pressure curves were applied at the inlet and outlet sections, respectively (Figures 5a and $6 \mathrm{a}$ ). The external parts were used to ensure a zero-pressure condition outside the aorta. The blood was modeled as a Newtonian fluid, with a density of $1060 \mathrm{~kg} / \mathrm{m}^{3}$ and a dynamic viscosity of $3.5 \mathrm{cP}$ [80]. During the first $0.3 \mathrm{~s}$ of the simulation, when the device crimping took place, the blood was pressurized to reach the end-diastolic pressure of 50 and $55 \mathrm{mmHg}$ for patient A and B respectively. Finally, two cardiac cycles were simulated with $69.8 \mathrm{bpm}$ and $71.4 \mathrm{bpm}$ for patient $\mathrm{A}$ and $\mathrm{B}$ respectively.

\section{Results}

The results of the transcatheter aortic valves implantation are shown in terms of stress field on the aorta, native valve and calcifications, and contact pressure on the inner surface of the aorta due to the contact with the stent. The final configuration of the transcatheter aortic valves in the patients is qualitative compared with the corresponding post-implantation CT scan or angiography acquisition.

For the FSI simulations, results were shown at the mid- (t1) and peak- $(\mathrm{t} 2)$ systolic phase and at the mid- (t3) and peak- (t4) diastolic phase of the second cardiac cycle in terms of stress field on the device pericardium and blood velocity field. RV and EROA [81] were calculated by post-processing the simulations and compared with the post-implantation Doppler echocardiography prognosis for both patients.

Once the device had reached its released configuration in the aortic root, the final configuration of the stent was qualitatively checked with that acquired $i n-H$ after the implantation. In relation to case A, the comparison between post-operative CT-scan and the numerical model exhibited a good positioning of the device in the reconstructed patient (Figure $3 a-3 b$ ). For case B, the comparison was done with the image from the angiographic exam, showing a good qualitative positioning (Figure 4a-4b).

The contact pressure field was examined in the inner surface of the aorta roots to analyze the interaction between the self-expandable valve and the vessel. Indeed, the self-expandable valves remain anchored to the aorta as a result of the radial force that they exert to the inner vessel wall due to the shape memory material properties. For both cases, the areas with a nonzero contact pressure were located at the stent contact areas, with a maximum value of 0.37 
$\mathrm{MPa}$ for case A (Figure 3c) and $0.29 \mathrm{MPa}$ for case B (Figure 4c). The maximum pressure changed by a maximum of $5 \%$ during the cardiac cycle.

The first principal stress field was evaluated for all the patient-specific aortic root components. The maximum stress areas of the native leaflets resulted near the calcifications for both cases with a maximum value of $1.13 \mathrm{MPa}$ and $1.22 \mathrm{MPa}$ during the systolic phase and $0.95 \mathrm{MPa}$ and 1.17 MPa during diastole for case A (Figure 3d) and B (Figure 4d) respectively. The corresponding maximum values for the calcifications were $2.73 \mathrm{MPa}$ and $7.96 \mathrm{MPa}$ during systole and 2.34 MPa and 7.79 MPa during diastole for case A (Figure 3d) and B (Figure 4d) respectively. Nevertheless, it should be noticed that these maximum stress values were limited to the area of connection between the native leaflets and the calcification deposits. In fact, the mean stress values for case A and B on the valve were $0.23 \mathrm{MPa}$ and $0.24 \mathrm{MPa}$, whereas on the calcifications the mean stress reached $0.12 \mathrm{MPa}$ and $0.21 \mathrm{MPa}$ respectively. On the aortic roots, the stress field was rather different between case A and B. In fact, in case B, where the calcification obstruction prevented the full stent release, stress values were higher. However, similar behavior was recognized in both cases. The maximum stress areas were located where the contact with the stent and the calcifications took place, with a maximum value of $1.27 \mathrm{MPa}$ and 1.32 MPa during systole, and 0.64 MPa and 0.78 MPa during diastole for case A (Figure $3 \mathrm{e}$ ) and B (Figure 4e) respectively. Moreover, both cases showed unreliable stress results on the aorta next to its ends, where the boundary conditions were applied. In these figures, the section of the stent struts is also shown at different planes to better depict the release configuration of the device inside the patients' aorta. In general, the stent contacted the aorta only in its proximal (plane 2) and distal portion (plane 4), whereas in the central area of the Valsalva sinus contacts were not detected (plane 3).

The first principal stress field was also evaluated for both pericardium parts of the device, the leaflets and the skirt. In general, the maximum stress areas resulted close to the commissure lines, where the leaflets connect the skirt and where the skirt is attached to the metallic frame. The maximum-mean stress values on the pericardium leaflets were, for case A (Figure $5 \mathrm{~b}$ ) and case $\mathrm{B}$ (Figure 6b), 0.73-0.13 MPa and 1.07-0.14 MPa during systole and 0.82-0.24 MPa and 2.1-0.36 $\mathrm{MPa}$ during diastole respectively.

Regarding the hemodynamics results, the velocity field was evaluated in the second cardiac cycle. The maximum velocity curves obtained from the numerical models were overlapped to the post-operative Doppler traces (Figure 7a); the estimated maximum velocity values at the systolic peak were $2.3 \mathrm{~m} / \mathrm{s}$ in case A and $3.1 \mathrm{~m} / \mathrm{s}$ in case B, in agreement with the Doppler values. Regarding the case $A$, the velocity contour map in different planes (Figure $5 \mathrm{c}$ ) shows a mean central jet through the open leaflets during systole and a complete closure of the valve with two minor PVLs during diastole. On the other hand, case B (Figure 6c) was characterized by two jets during systole, the main through the open leaflets and the other one outside the valve, and by a complete closure of the valve with a main significant PVL during diastole. Blood flow rate curves were calculated for both cases (Figure 7b) and RV values were calculated by integrating the curves over the regurgitant period with a resulting value of 26.88 $\mathrm{mL}$ for case $\mathrm{A}$ and $43.73 \mathrm{~mL}$ for case B. Regurgitant jet time-velocities were calculated by integrating the regurgitant velocity over the regurgitant period and the consequent EROA values resulted of $0.097 \mathrm{~cm}^{2}$ for case A and $0.146 \mathrm{~cm}^{2}$ for case B.

\section{Discussion}


Despite TAVR has been increasingly used in the last twenty years, many factors affecting the vasculature-stent interaction are still unknown and the procedure is not free of complications and non-predictable adverse events [2]. Each patient has a different anatomy and morphology, but also a different clinical course which makes the TAVR outcomes hard to predict. In this context, numerical analyses are a powerful tool able to obtain some guidelines in the preoperative stage. In particular, the presence of structures that interact with blood entails the FSI method to be the best numerical analysis procedure $[28,58]$. In this study, we modeled the TAVR procedure of two real clinical cases by a strong, two-way, segregated FSI algorithm. A high-fidelity CAD model of the device was created, and patient-specific domains were segmented from preoperative CT scans. Once the appropriate discretization of all the components was done, the different material properties were modeled with the most advanced constitutive laws from the literature, based on the available experimental data. All the steps of the procedure were considered, including the pre-dilatation of the stenotic native valve, the crimping of the device and its in situ release through a catheter pulling-out maneuver. Patientspecific pressure curves were applied as boundary conditions to the FSI simulations to reproduce a highly realistic response of the device. In the literature, several numerical studies on TAVR can be found, most of which were here reported and classified. To the best of our knowledge, the novelty of this work is precisely the segregated 2-ways FSI methodology, which combines the main strengths of the structural and CFD analyses. It constitutes a novelty also compared to the few previous FSI studies [43,48,54], especially regarding the patientspecific domain modeling. For all these reasons, the presented model provided useful results to investigate the main complications after TAVR.

From a structural viewpoint, the in-silico model allows investigating the optimal positioning of the device, as well as to study other complications related to the intervention such as device migration and atrioventricular block [18]. The modeling of the anisotropic hyperelastic nature of the aorta [55], the native valve and the calcifications [30,37] provides an accurate representation of the release phase of the device. Further, considering the pericardium device components during the release phase, it has already been proven to be important when evaluating the performance of the device [40]. Moreover, the inclusion of the pericardium parts in the crimping phase allows estimating the stress field on the leaflets to investigate their degeneration and, therefore, their long-term durability [25]. The comparison of the two different patients, case $\mathrm{A}$ and $\mathrm{B}$, with different calcification configurations, demonstrated also how the calcification location and size influence the final configurations of the device and its eccentric distortion [19]. Calcifications also influence the stress field on the leaflets, which resulted maximum near the commissure tips, as in a previous work [34]. The maximum stress value on the leaflets resulted higher in case $B$, in which the calcifications were bigger. This result suggests the existence of an optimal orientation of the device with respect the patientspecific calcification configuration [49]. The potential vascular damage could be evaluated from the stress field, as well as from the pressure contact area coming from the radial forces that the device exerts on the vessel. The entities of the stress distributions resulted appropriately comparable with a previous structural study [38], as well as the contact pressure [56]. The contact pressures can also be used as a surrogate quantity to evaluate potential device migration problems.

Moreover, the main hemodynamic complication after the procedure is the presence of leaks during diastole. Some evaluations of this problems have already been performed using structural simulations. These evaluations indicate a correlation between the final configuration 
after implantation and the presence of PVL [46,82]. CFD studies, following or not structural analyses, were also carried out to quantify PVL [45,53]. In this regard, the FSI modeling provides a unified methodology, enabling the investigation of structural aspects and the quantification of hemodynamic quantities at the same time. The calculation of velocity fields and flow rate curves allows the quantification of the most common clinical parameters used to assess the presence of PVL, such as the regurgitation volume and the EROA [8]. These parameters, calculated for both the considered patients, confirmed the postoperative prognosis of mild and moderate PVL. In particular, we had selected these two patients especially because presented different outcomes with the same implanted valve and this allowed us to show the versatility of the methodology and its ability to represent different situations.

Limitations

Our FSI simulations are not absent from limitations. The assumption of linear elasticity to model the porcine pericardium is justified by the restricted working strain range and by its weak hyperelastic behavior [83]. However, a more sophisticated hyperelastic material might be incorporated. Pre-stress field on the aorta should be included in future works to replicate the real end-diastole configurations of patients, especially in works focused on arterial wall damage induced by TAVR. Moreover, data from pathological aortic root should be considered for the material properties of the patient domain. A longer model of the segmented aorta should be also considered.

Regarding the fluid domain, the inclusion of the coronary arteries could give additional indications to better foresee clinical outcomes. Indeed, the inclusion of the coronary arteries with appropriate Windkessel-type boundary conditions [54] may be included in future FSI models. Furthermore, turbulence model was not considered in this work. Finally, an important limitation of this model is its high computational cost. Indeed, even if this kind of simulation cannot claim, nowadays, to be real-time in the clinical application as its duration is around 7 days (computer node with 4 CPU Xeon E5-4627 v3 with 10 cores per CPU and 256 GB of RAM memory). In the future, this limitation can be tackled by adopting different modeling techniques, such as reducing the complexity of the stent with beam elements.

\section{Conclusion}

In conclusion, in this study the potentiality of the proposed methodology in terms of available results by reproducing two real clinical outcomes after TAVR implantation was shown. The comparison between the calculated, not estimate, PVL and the clinical diagnosis is taken as an example to show the impact of this work. This kind of numerical methodology is, in our opinion, very useful to guide clinical decision making before and after the procedure.

\section{Compliance with Ethical Standards}

Author Luraghi, Author Migliavacca, Author García-González, Author Chiastra, Author Rossi, Author Cao, Author Stefanini and Author Rodriguez Matas declare that they have no conflict of interest.

No human or animal studies were carried out by the authors for this article. Institutional review board approval was obtained from Humanitas University for sharing of the anonymized patient images and data. 


\section{References}

1. Smith CR, Leon MB, Mack MJ, Miller DC, Moses JW, Svensson LG, et al. Transcatheter versus surgical aortic-valve replacement in high-risk patients. N Engl J Med. 2011;364:218798.

2. Reardon MJ, Van Mieghem NM, Popma JJ, Kleiman NS, Søndergaard L, Mumtaz M, et al. Surgical or Transcatheter Aortic-Valve Replacement in Intermediate-Risk Patients. N Engl J Med. 2017;376:1321-31.

3. Mahtta D, Elgendy IY, Bavry AA. From CoreValve to Evolut PRO: Reviewing the Journey of Self-Expanding Transcatheter Aortic Valves. Cardiol Ther. 2017;6:183-92.

4. Grube E, Laborde JC, Gerckens U, Felderhoff T, Sauren B, Buellesfeld L, et al. Percutaneous Implantation of the CoreValve Self-Expanding Valve Prosthesis in High-Risk Patients With Aortic Valve Disease: The Siegburg First-in-Man Study. Circulation. 2006;114:1616-24.

5. Généreux P, Head SJ, Hahn R, Daneault B, Kodali S, Williams MR, et al. Paravalvular Leak After Transcatheter Aortic Valve Replacement. J Am Coll Cardiol. 2013;61:1125-36.

6. Morgan RB, Kwong RY. When Accurate Flow Quantitation Matters. J Am Coll Cardiol. 2016;68:586-8.

7. Vy P, Auffret V, Badel P, Rochette M, Le Breton H, Haigron P, et al. Review of patientspecific simulations of transcatheter aortic valve implantation. Int J Adv Eng Sci Appl Math. 2016;8:2-24.

8. Kappetein AP, Head SJ, Genereux P, Piazza N, Van Mieghem NM, Blackstone EH, et al. Updated standardized endpoint definitions for transcatheter aortic valve implantation: the Valve Academic Research Consortium-2 consensus document (VARC-2). Eur J CardioThoracic Surg. 2012;42:S45-60.

9. Dasi LP, Hatoum H, Kheradvar A, Zareian R, Alavi SH, Sun W, et al. On the Mechanics of Transcatheter Aortic Valve Replacement. Ann Biomed Eng. 2017;45:310-31.

10. Abdelghani M, Miyazaki Y, De Boer E, Aben J-P, Van Sloun M, Suchecki T, et al. Videodensitometric quantification of paravalvular regurgitation of a transcatheter aortic valve: in vitro validation. EuroIntervention. 2018;13:1527-35.

11. Schäfer T, Doose C, Fujita B, Utzenrath M, Egron S, Schmitz C, et al. Preclinical determination of the best functional position for transcatheter heart valves implanted in rapid deployment bioprostheses. EuroIntervention. 2017;12:1706-14.

12. Laflamme J, Puri R, Urena M, Laflamme L, DeLarochellière H, Abdul-Jawad Altisent O, et al. Incidence and risk factors of hemolysis after transcatheter aortic valve implantation with a balloon-expandable valve. Am J Cardiol. 2015;115:1574-9.

13. Marwan M, Mekkhala N, Göller M, Röther J, Bittner D, Schuhbaeck A, et al. Leaflet thrombosis following transcatheter aortic valve implantation. J Cardiovasc Comput Tomogr. 2018;12:8-13.

14. Testa L, Latib A. Assessing the Risk of Leaflet Motion Abnormality Following Transcatheter Aortic Valve Implantation. Interv Cardiol Rev. 2018;13:37-9.

15. Laschinger JC, Wu C, Ibrahim NG, Shuren JE. Reduced Leaflet Motion in Bioprosthetic Aortic Valves - The FDA Perspective. N Engl J Med. 2015;373:1996-8.

16. Ducci A, Pirisi F, Tzamtzis S, Burriesci G. Transcatheter aortic valves produce unphysiological flows which may contribute to thromboembolic events: An in-vitro study. J Biomech. 2016;49:4080-9.

17. Bianchi M, Marom G, Ghosh RP, Fernandez HA, Taylor JR, Slepian MJ, et al. Effect of Balloon-Expandable Transcatheter Aortic Valve Replacement Positioning: A Patient-Specific Numerical Model. Artif Organs. 2016;40:E292-304.

18. Siontis GCM, Jüni P, Pilgrim T, Stortecky S, Büllesfeld L, Meier B, et al. Predictors of 
Permanent Pacemaker Implantation in Patients With Severe Aortic Stenosis Undergoing TAVR: A Meta-Analysis. J Am Coll Cardiol. 2014;64:129-40.

19. Gunning PS, Saikrishnan N, Yoganathan AP, McNamara LM. Total ellipse of the heart valve: the impact of eccentric stent distortion on the regional dynamic deformation of pericardial tissue leaflets of a transcatheter aortic valve replacement. J R Soc Interface. 2015; 12(113):20150737.

20. Zenses A-S, Evin MA, Stanová V, Clavel M-A, Barragan P, Rodés-Cabau J, et al. Effect of size and position of self-expanding transcatheter valve on haemodynamics following valvein-valve procedure in small surgical bioprostheses: an in vitro study. EuroIntervention. 2018;14:e282-9.

21. Stoller M, Gloekler S, Zbinden R, Tueller D, Eberli F, Windecker S, et al. Left ventricular afterload reduction by transcatheter aortic valve implantation in severe aortic stenosis and its prompt effects on comprehensive coronary haemodynamics. EuroIntervention. 2018;14:16673.

22. Jung C, Lichtenauer M, Figulla H-R, Wernly B, Goebel B, Foerster M, et al. Microparticles in patients undergoing transcatheter aortic valve implantation (TAVI). Heart Vessels. 2017;32:458-66.

23. Li K, Sun W. Simulated Thin Pericardial Bioprosthetic Valve Leaflet Deformation Under Static Pressure-Only Loading Conditions: Implications for Percutaneous Valves. Ann Biomed Eng. 2010;38:2690-701.

24. Tzamtzis S, Viquerat J, Yap J, Mullen MJ, Burriesci G. Numerical analysis of the radial force produced by the Medtronic-CoreValve and Edwards-SAPIEN after transcatheter aortic valve implantation (TAVI). Med Eng Phys. 2013;35:125-30.

25. Abbasi M, Azadani AN. Leaflet stress and strain distributions following incomplete transcatheter aortic valve expansion. J Biomech. 2015;48:3663-71.

26. Martin C, Sun W. Comparison of transcatheter aortic valve and surgical bioprosthetic valve durability: A fatigue simulation study. J Biomech. 2015;48:3026-34.

27. Li K, Sun W. Simulated transcatheter aortic valve deformation: A parametric study on the impact of leaflet geometry on valve peak stress. Int J Numer Method Biomed Eng. 2016; 02814 .

28. Mao W, Li K, Sun W. Fluid-Structure Interaction Study of Transcatheter Aortic Valve Dynamics Using Smoothed Particle Hydrodynamics. Cardiovasc Eng Technol. 2016;7:37488.

29. Martin C, Sun W. Transcatheter Valve Underexpansion Limits Leaflet Durability: Implications for Valve-in-Valve Procedures. Ann Biomed Eng. 2016;

30. Sturla F, Ronzoni M, Vitali M, Dimasi A, Vismara R, Preston-Maher G, et al. Impact of different aortic valve calcification patterns on the outcome of transcatheter aortic valve implantation: A finite element study. J Biomech. 2016;49:2520-30.

31. Hopf R, Sündermann SH, Born S, Ruiz CE, Van Mieghem NM, De Jaegere PP, et al. Postoperative analysis of the mechanical interaction between stent and host tissue in patients after transcatheter aortic valve implantation. J Biomech. 2017;53:15-21.

32. Vahidkhah K, Javani S, Abbasi M, Azadani PN, Tandar A, Dvir D, et al. Blood Stasis on Transcatheter Valve Leaflets and Implications for Valve-in-Valve Leaflet Thrombosis. Ann Thorac Surg. 2017;104:751-9.

33. Wald S, Liberzon A, Avrahami I. A numerical study of the hemodynamic effect of the aortic valve on coronary flow. Biomech Model Mechanobiol. 2018;17:319-38.

34. Xuan Y, Krishnan K, Ye J, Dvir D, Guccione JM, Ge L, et al. Stent and Leaflet Stresses in 29-mm Second-Generation Balloon-Expandable Transcatheter Aortic Valve. Ann Thorac Surg. 2017;104:773-81.

35. Capelli C, Bosi GM, Cerri E, Nordmeyer J, Odenwald T, Bonhoeffer P, et al. Patient- 
specific simulations of transcatheter aortic valve stent implantation. Med Biol Eng Comput. 2012;50:183-92.

36. Auricchio F, Conti M, Morganti S, Reali A. Simulation of transcatheter aortic valve implantation: a patient-specific finite element approach. Comput Methods Biomech Biomed Engin. 2014;17:1347-57.

37. Russ C, Hopf R, Hirsch S, Sundermann S, Falk V, Szekely G, et al. Simulation of transcatheter aortic valve implantation under consideration of leaflet calcification. 2013 35th Annu Int Conf IEEE Eng Med Biol Soc. 2013. p. 711-4.

38. Gunning PS, Vaughan TJ, McNamara LM. Simulation of self expanding transcatheter aortic valve in a realistic aortic root: implications of deployment geometry on leaflet deformation. Ann Biomed Eng. 2014;42:1989-2001.

39. Morganti S, Conti M, Aiello M, Valentini A, Mazzola A, Reali A, et al. Simulation of transcatheter aortic valve implantation through patient-specific finite element analysis: Two clinical cases. J Biomech. 2014;47:2547-55.

40. Bailey J, Curzen N, Bressloff NW. Assessing the impact of including leaflets in the simulation of TAVI deployment into a patient-specific aortic root. Comput Methods Biomech Biomed Engin. 2016;19:733-44.

41. Kopanidis A, Pantos I, Alexopoulos N, Theodorakakos A, Efstathopoulos E, Katritsis D. Aortic Flow Patterns After Simulated Implantation of Transcatheter Aortic Valves. Hellenic J Cardiol. 2015;56:418-28.

42. Wang Q, Kodali S, Primiano C, Sun W. Simulations of transcatheter aortic valve implantation: implications for aortic root rupture. Biomech Model Mechanobiol. 2015;14:29_ 38 .

43. Basri AA, Zuber M, Zakaria MS, Basri EI, Aziz AFA, Ali RM, et al. The Hemodynamic Effects of Paravalvular Leakage Using Fluid Structure Interaction; Transcatheter Aortic Valve Implantation Patient. J Med Imaging Heal Informatics. 2016;6:1513-8.

44. Bosmans B, Famaey N, Verhoelst E, Bosmans J, Vander Sloten J. A validated methodology for patient specific computational modeling of self-expandable transcatheter aortic valve implantation. J Biomech. 2016;49:2824-30.

45. De Jaegere P, De Santis G, Rodriguez-Olivares R, Bosmans J, Bruining N, Dezutter T, et al. Patient-Specific Computer Modeling to Predict Aortic Regurgitation After Transcatheter Aortic Valve Replacement. JACC Cardiovasc. Interv. 2016. p. 508-12.

46. Morganti S, Brambilla N, Petronio AS, Reali A, Bedogni F, Auricchio F. Prediction of patient-specific post-operative outcomes of TAVI procedure: The impact of the positioning strategy on valve performance. J Biomech. 2016;49:2513-9.

47. Ovcharenko EA, Klyshnikov KU, Yuzhalin AE, Savrasov GV, Kokov AN, Batranin AV, et al. Modeling of transcatheter aortic valve replacement: Patient specific vs general approaches based on finite element analysis. Comput Biol Med. 2016;69:29-36.

48. Wu W, Pott D, Mazza B, Sironi T, Dordoni E, Chiastra C, et al. Fluid-Structure Interaction Model of a Percutaneous Aortic Valve: Comparison with an In Vitro Test and Feasibility Study in a Patient-Specific Case. Ann Biomed Eng. 2016;44:590-603.

49. Bailey J, Curzen N, Bressloff NW. The impact of imperfect frame deployment and rotational orientation on stress within the prosthetic leaflets during transcatheter aortic valve implantation. J Biomech. 2017;53:22-8.

50. Cabrera MS, Oomens CWJ, Baaijens FPT. Understanding the requirements of selfexpandable stents for heart valve replacement: Radial force, hoop force and equilibrium. J Mech Behav Biomed Mater. 2017;68:252-64.

51. El Faquir N, Ren B, Van Mieghem NM, Bosmans J, De Jaegere PP. Patient-specific computer modelling - its role in the planning of transcatheter aortic valve implantation. Neth Heart J. 2017;25:100-5. 
52. Bosi GM, Capelli C, Cheang MH, Delahunty N, Mullen M, Taylor AM, et al. Populationspecific material properties of the implantation site for transcatheter aortic valve replacement finite element simulations. J Biomech. 2018;71:236-44.

53. Mao W, Wang Q, Kodali S, Sun W. Numerical Parametric Study of Paravalvular Leak Following a Transcatheter Aortic Valve Deployment Into a Patient-Specific Aortic Root. J Biomech Eng. 2018;140:101007.

54. Kandail HS, Trivedi SD, Shaikh AC, Bajwa TK, O'Hair DP, Jahangir A, et al. Impact of annular and supra-annular CoreValve deployment locations on aortic and coronary artery hemodynamics. J Mech Behav Biomed Mater. 2018;86:131-42.

55. Finotello A, Morganti S, Auricchio F. Finite element analysis of TAVI: Impact of native aortic root computational modeling strategies on simulation outcomes. Med Eng Phys. 2017;47:2-12.

56. Rocatello G, El Faquir N, De Santis G, Iannaccone F, Bosmans J, De Backer O, et al. Patient-Specific Computer Simulation to Elucidate the Role of Contact Pressure in the Development of New Conduction Abnormalities After Catheter-Based Implantation of a SelfExpanding Aortic Valve. Circ Cardiovasc Interv. 2018;11:e005344.

57. Bianchi M, Marom G, Ghosh RP, Rotman OM, Parikh P, Gruberg L, et al. Patient-specific simulation of transcatheter aortic valve replacement: impact of deployment options on paravalvular leakage. Biomech Model Mechanobiol. 2019;18:435-51.

58. Luraghi G, Wu W, De Gaetano F, Rodriguez Matas JF, Moggridge GD, Serrani M, et al. Evaluation of an aortic valve prosthesis: Fluid-structure interaction or structural simulation? J Biomech. 2017;58:45-51.

59. Ghosh RP, Marom G, Rotman OM, Slepian MJ, Prabhakar S, Horner M, et al. Comparative Fluid-Structure Interaction Analysis of Polymeric Transcatheter and Surgical Aortic Valves' Hemodynamics and Structural Mechanics. J Biomech Eng. 2018;140:121002.

60. Wei ZA, Sonntag SJ, Toma M, Singh-Gryzbon S, Sun W. Computational Fluid Dynamics Assessment Associated with Transcatheter Heart Valve Prostheses: A Position Paper of the ISO Working Group. Cardiovasc Eng Technol. 2018;9:289-99.

61. Puso MA. A highly efficient enhanced assumed strain physically stabilized hexahedral element. Int J Numer Methods Eng. 2000;49:1029-64.

62. Belytschko T, Lin JI, Chen-Shyh T. Explicit algorithms for the nonlinear dynamics of shells. Comput Methods Appl Mech Eng. 1984;42:225-51.

63. Kosloff D, Frazier GA. Treatment of hourglass patterns in low order finite element codes. Int J Numer Anal Methods Geomech. 1978;2:57-72.

64. Luraghi G, Migliavacca F, Rodriguez Matas JF. Study on the Accuracy of Structural and FSI Heart Valves Simulations. Cardiovasc Eng Technol. 2018;9:1-16.

65. Belytschko T, Tsay CS, Liu WK. A stabilization matrix for the bilinear mindlin plate element. Comput Methods Appl Mech Eng. 1981;29:313-27.

66. Martin C, Pham T, Sun W. Significant differences in the material properties between aged human and porcine aortic tissues. Eur J Cardio-Thoracic Surg. 2011;40:28-34.

67. Bavo AM, Rocatello G, Iannaccone F, Degroote J, Vierendeels J, Segers P. Fluid-Structure Interaction Simulation of Prosthetic Aortic Valves: Comparison between Immersed Boundary and Arbitrary Lagrangian-Eulerian Techniques for the Mesh Representation. PLoS One. 2016;11:e0154517.

68.https://www.medtronic.com/us-

en/healthcareprofessionals/products/cardiovascular/transcatheter-aortic-heart-valves/evolut-

pro.html

69. Nagtegaal JC, Parks DM, Rice JR. On numerically accurate finite element solutions in the fully plastic range. Comput Methods Appl Mech Eng. 1974;4:153-77.

70. Holzapfel GA, Ogden RW. Constitutive modelling of passive myocardium: a structurally 
based framework for material characterization. Philos Trans R Soc A Math Phys Eng Sci. 2009;367:3445-75.

71. Riveros F, Chandra S, Finol EA, Gasser TC, Rodriguez JF. A Pull-Back Algorithm to Determine the Unloaded Vascular Geometry in Anisotropic Hyperelastic AAA Passive Mechanics. Ann Biomed Eng. 2013;41:694-708.

72. Vande Geest JP, Sacks MS, Vorp DA. The effects of aneurysm on the biaxial mechanical behavior of human abdominal aorta. J Biomech. 2006;39:1324-34.

73. Driessen NJ, Boerboom RA, Huyghe JM, Bouten C V, Baaijens FP. Computational analyses of mechanically induced collagen fiber remodeling in the aortic heart valve. $\mathrm{J}$ Biomech Eng. 2003;125:549-57.

74. Alastrué V, Garía A, Peña E, Rodríguez JF, Martínez MA, Doblaré M. Numerical framework for patient-specific computational modelling of vascular tissue. Int $\mathrm{J}$ Numer Method Biomed Eng. 2010;26:35-51.

75. Holzapfel GA, Gasser TC, Ogden RW. A New Constitutive Framework for Arterial Wall Mechanics and a Comparative Study of Material Models. J Elast. 2000;61:1-48.

76. Holzapfel GA, Sommer G, Regitnig P. Anisotropic mechanical properties of tissue components in human atherosclerotic plaques. J Biomech Eng. 2004;126:657-65.

77. Morlacchi S, Chiastra C, Gastaldi D, Pennati G, Dubini G, Migliavacca F. Sequential Structural and Fluid Dynamic Numerical Simulations of a Stented Bifurcated Coronary Artery. J Biomech Eng. 2011;133:121010.

78. Mummert J, Sirois E, Sun W. Quantification of Biomechanical Interaction of Transcatheter Aortic Valve Stent Deployed in Porcine and Ovine Hearts. Ann Biomed Eng. 2013;41:577-86. 79. Marom G. Numerical Methods for Fluid-Structure Interaction Models of Aortic Valves. Arch Comput Methods Eng. 2015;22:595-620.

80. Mao W, Caballero A, McKay R, Primiano C, Sun W. Fully-coupled fluid-structure interaction simulation of the aortic and mitral valves in a realistic 3D left ventricle model. PLoS One. 2017;12:e0184729.

81. Enriquez-Sarano M, Seward JB, Bailey KR, Tajik AJ. Effective regurgitant orifice area: a noninvasive Doppler development of an old hemodynamic concept. J Am Coll Cardiol. 1994;23:443-51.

82. Sirois E, Mao W, Li K, Calderan J, Sun W. Simulated Transcatheter Aortic Valve Flow: Implications of Elliptical Deployment and Under-Expansion at the Aortic Annulus. Artif Organs. 2018;42:E141-52.

83. Martin C, Pham T, Sun W. Significant differences in the material properties between aged human and porcine aortic tissues. Eur J Cardiothorac Surg. 2011;40:28-34.

\section{Captions}

Figure 1: Numerical model of the CoreValve Evolute R (Medtronic): (a) CAD model of the constant-diameter stent; (b) final configuration of the device with stent (gray) and pericardium tissue (brown); (c) detail of the numerical grids of the stent (hexahedral solid elements in grey), the leaflets (quadrilateral shell elements in brown) and the skirt (triangular shell elements in brown); (d) kinematics of implantation of the transcatheter aortic valve (TAV) by catheter lifting, from the crimped to the released configuration.

Figure 2: Image-based aorta models: (a) principal directions of stress obtained through a preanalysis on the aorta; the two arrows highlight the first $\underline{\mathbf{a}}$ and the second $\underline{\mathbf{b}}$ principal directions, based on which the local coordinate systems are defined. (b) Computed local directions for the model; the longitudinal directions are marked in red, whereas the axial ones are marked in blue. 
(c-d) Reconstructed aorta model of patients A and B respectively: (left) complete view with the native valve and calcification, (center) frontal view of the native valve with the reconstructed calcifications; (right) fluid domain discretized with hexahedral elements where the structures of each patient are immersed in.

Figure 3: Structural results of Case A: Post-operative CT coronal plane scan without (a) and with (b) the numerical model overlapped. (c) Contour map of the contact pressure (MPa) on the aorta inner surface due to the device contact at the beginning of the cardiac cycle; (d) contour map of the first principal stress (MPa) in the native valve and calcifications and (e) in four planes of the aorta at the beginning of the systolic phase.

Figure 4: Structural results of Case B: Post-operative angiography without (a) and with (b) the numerical model overlapped. Contour map of the contact pressure $(\mathrm{MPa})$ on the aorta inner surface due to the device contact at the beginning of the cardiac cycle; (d) contour map of the first principal stress $(\mathrm{MPa})$ in the native valve and calcifications and (e) in four planes of the aorta at the beginning of the systolic phase.

Figure 5: Results of Case A: (a) Patient-specific pressure curves imposed as boundary conditions at the inlet and outlet sections of the fluid domain (1) with the time points where results are analyzed. (b) Contour maps of the first principal stress in the pericardium parts of the device. (c) Contours of velocity magnitude in four planes of the blood domain.

Figure 6: Results of Case B: (a) Patient-specific pressure curves imposed as boundary conditions at the inlet and outlet sections of the fluid domain (1) with the time points where results are analyzed. (b) Contour maps of the first principal stress in the pericardium parts of the device. (c) Contours of velocity magnitude in four planes of the blood domain.

Figure 7: PVL estimation: (a) post-operative Doppler trace for case A and B with overlapped (yellow) maximum velocity curves from the numerical model; (b) blood flow rate curves from the numerical model for case A and B and the computed RV and EROA. 


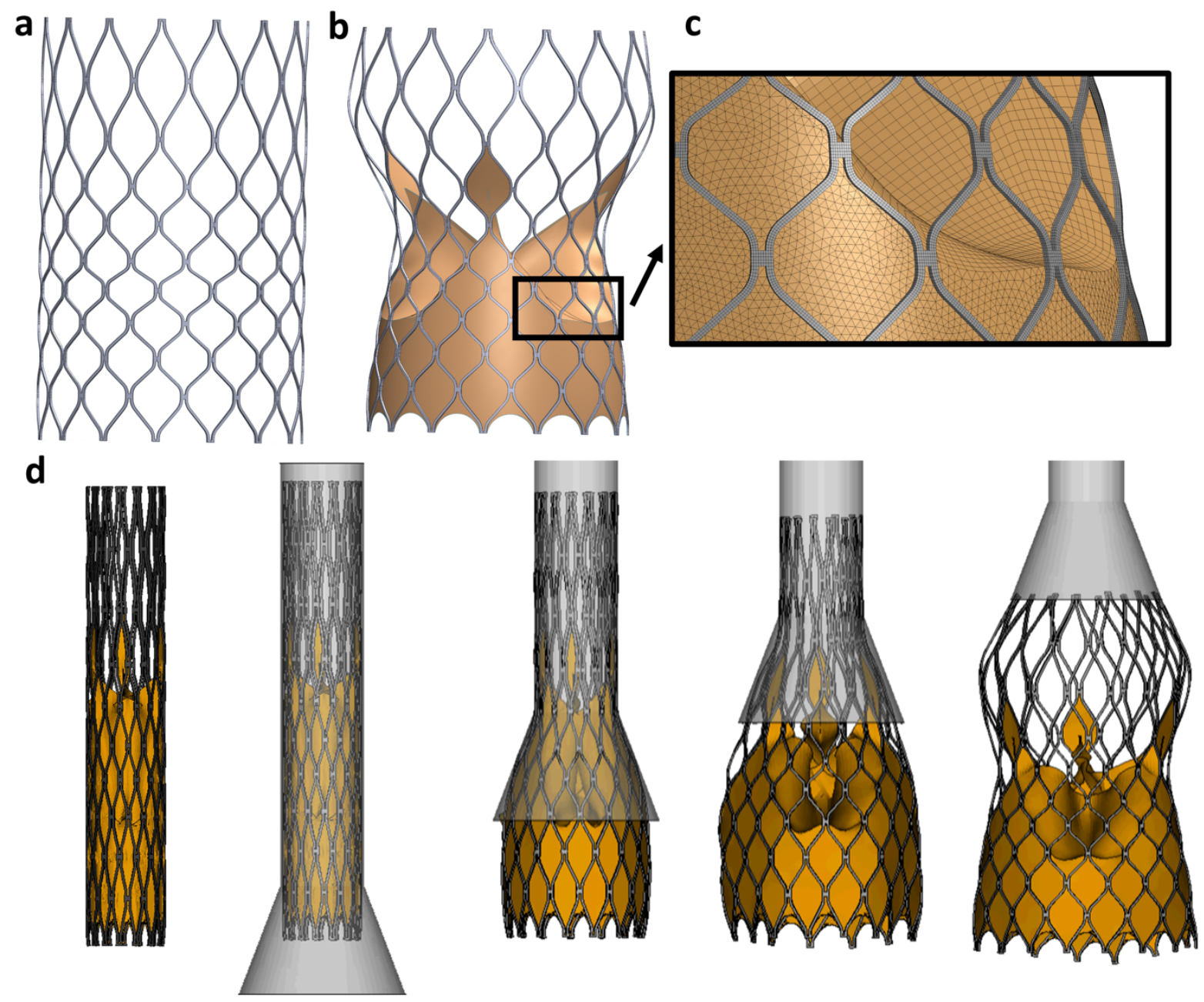

Figure 1 


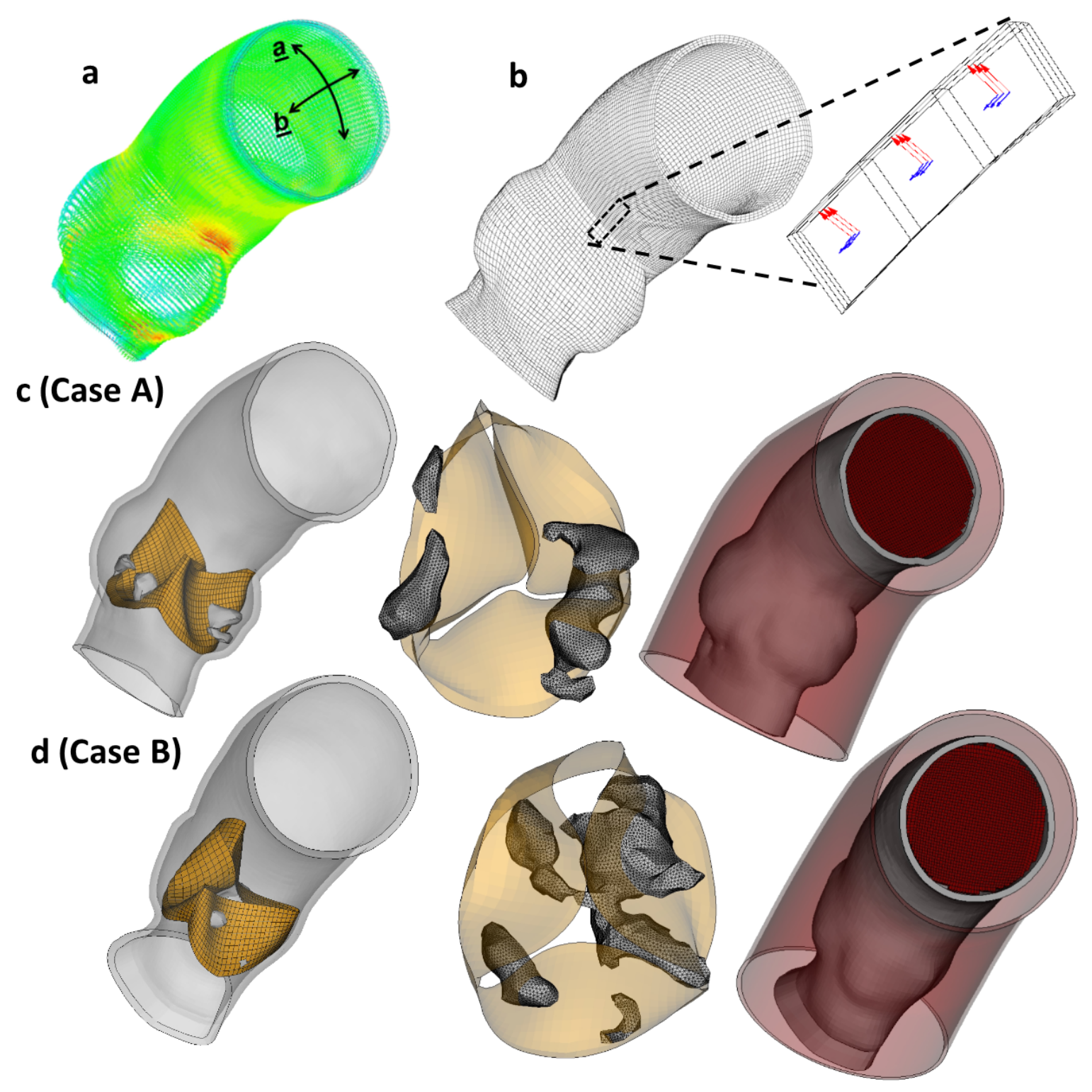

Figure 2 

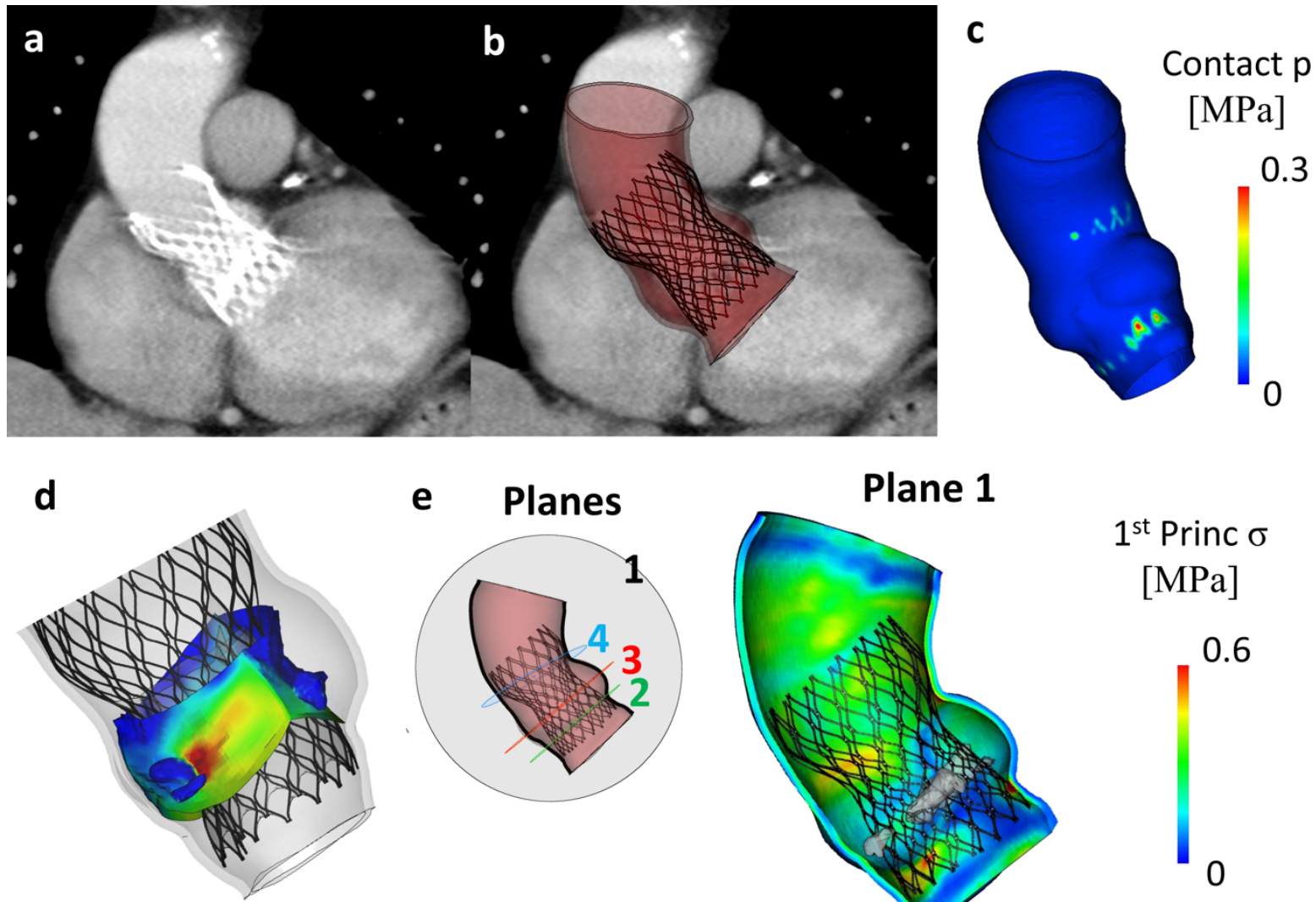

Plane 1

Plane 2
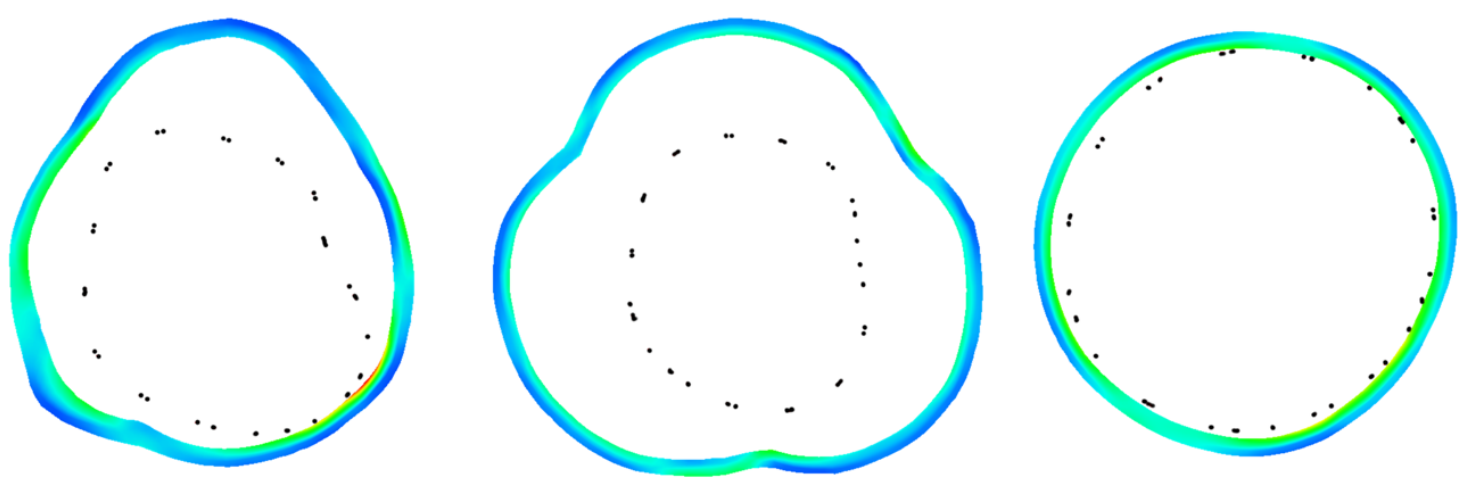

Figure 3 

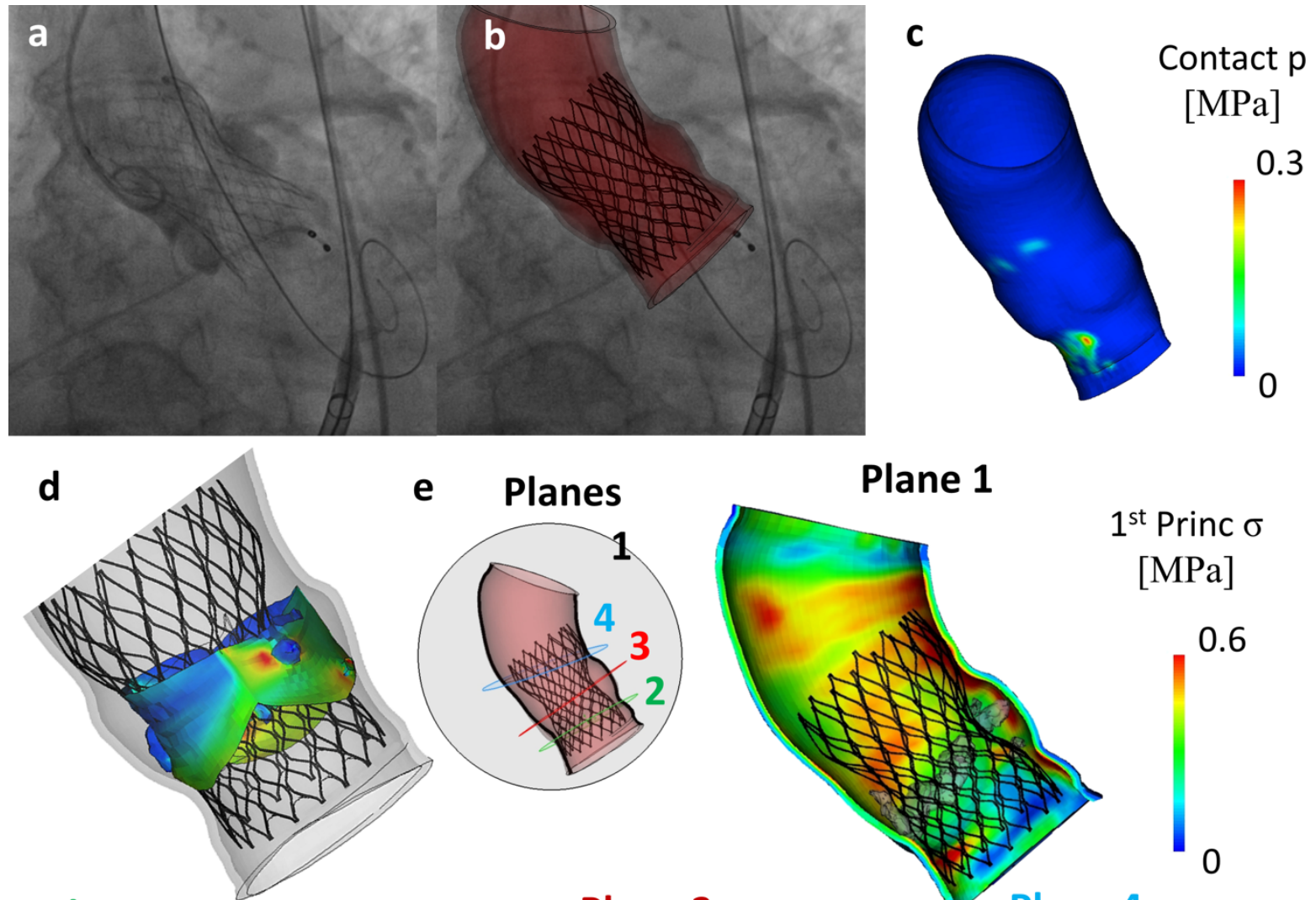

e Planes

Plane 2

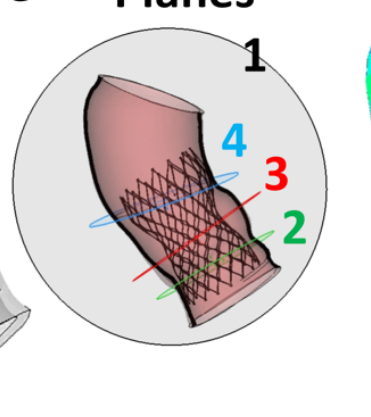

Plane 1

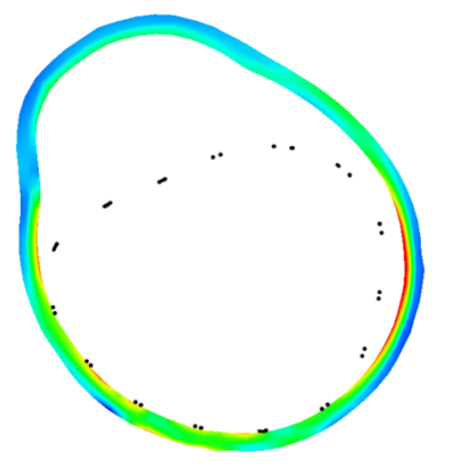

Plane 3
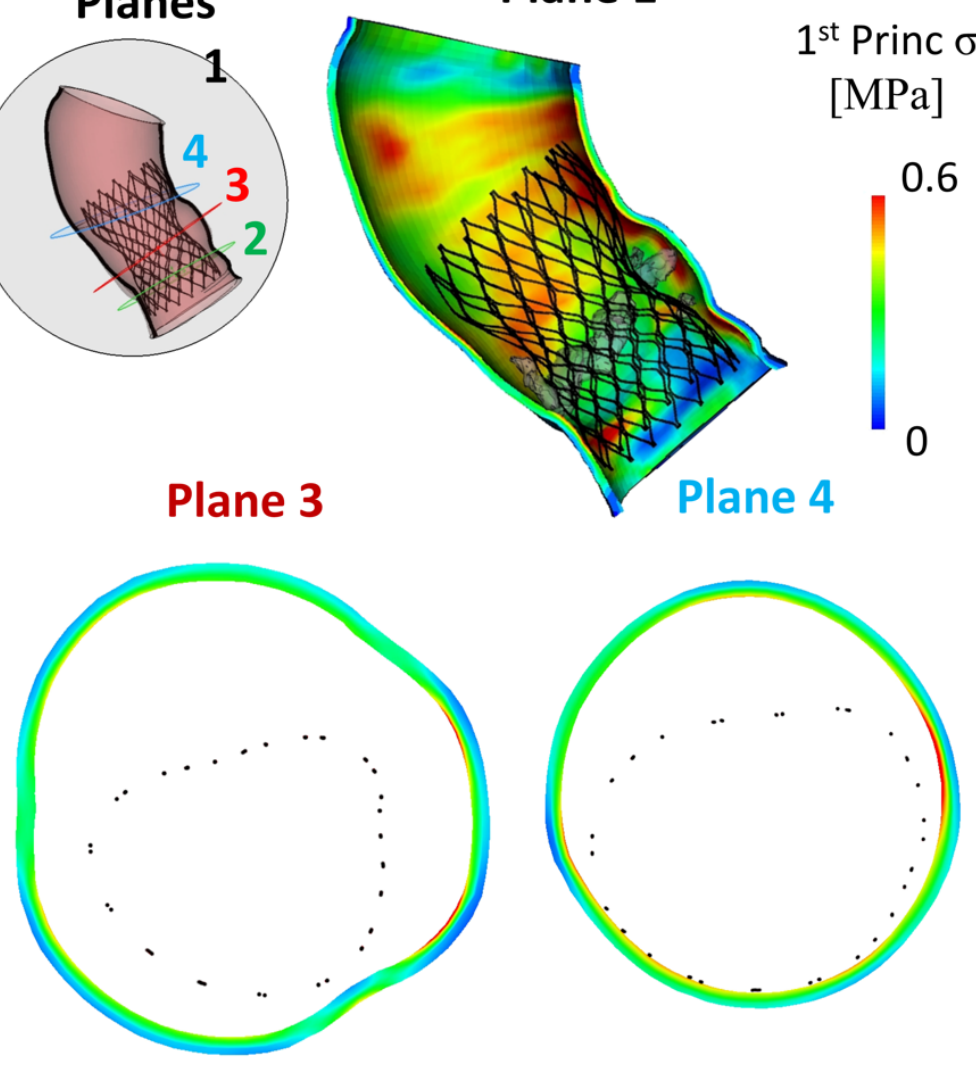

Figure 4 

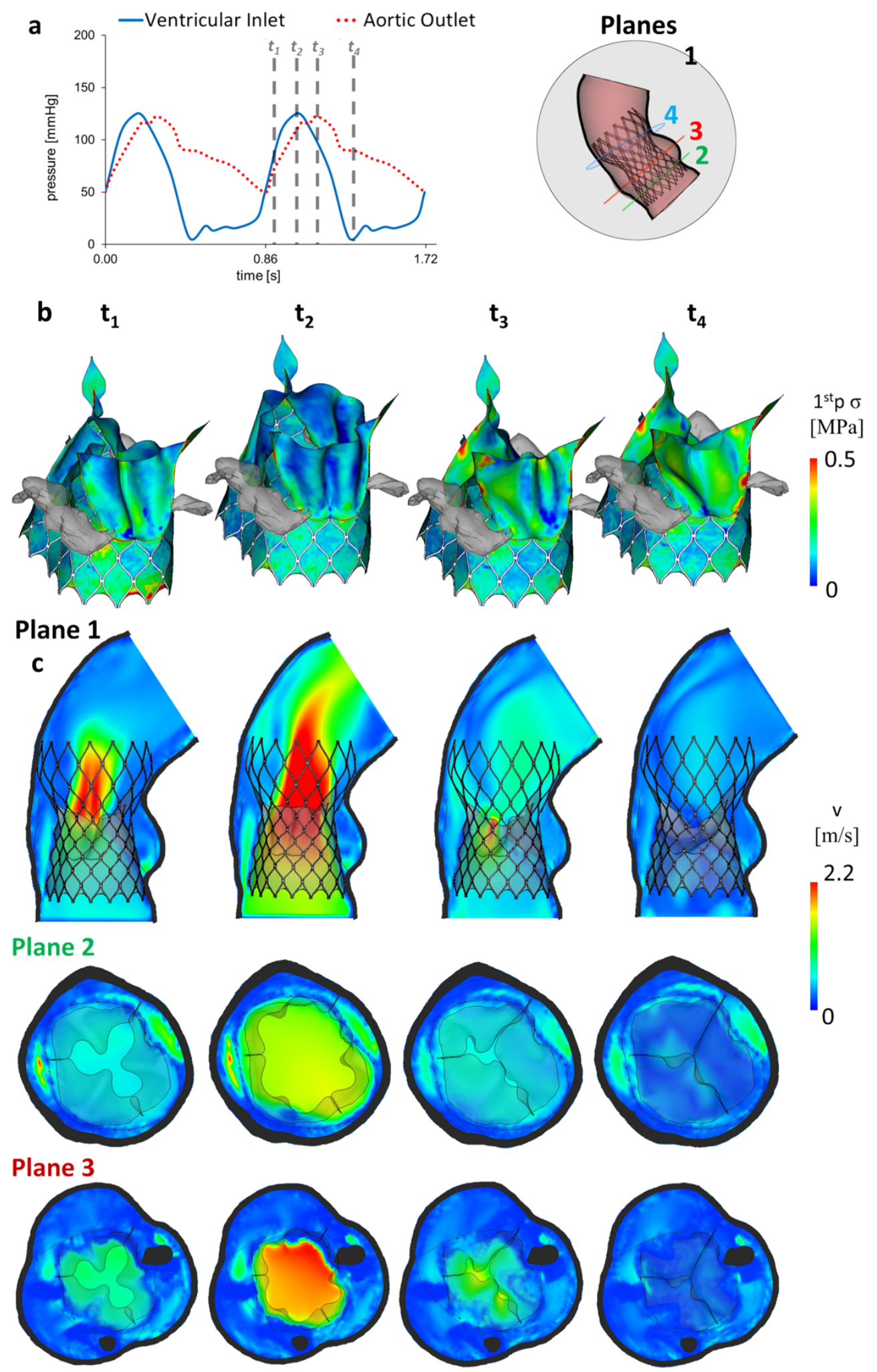

Plane 4
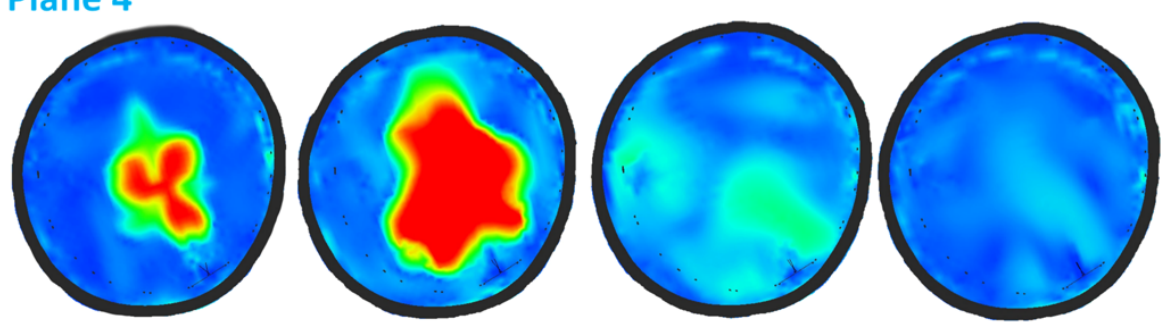

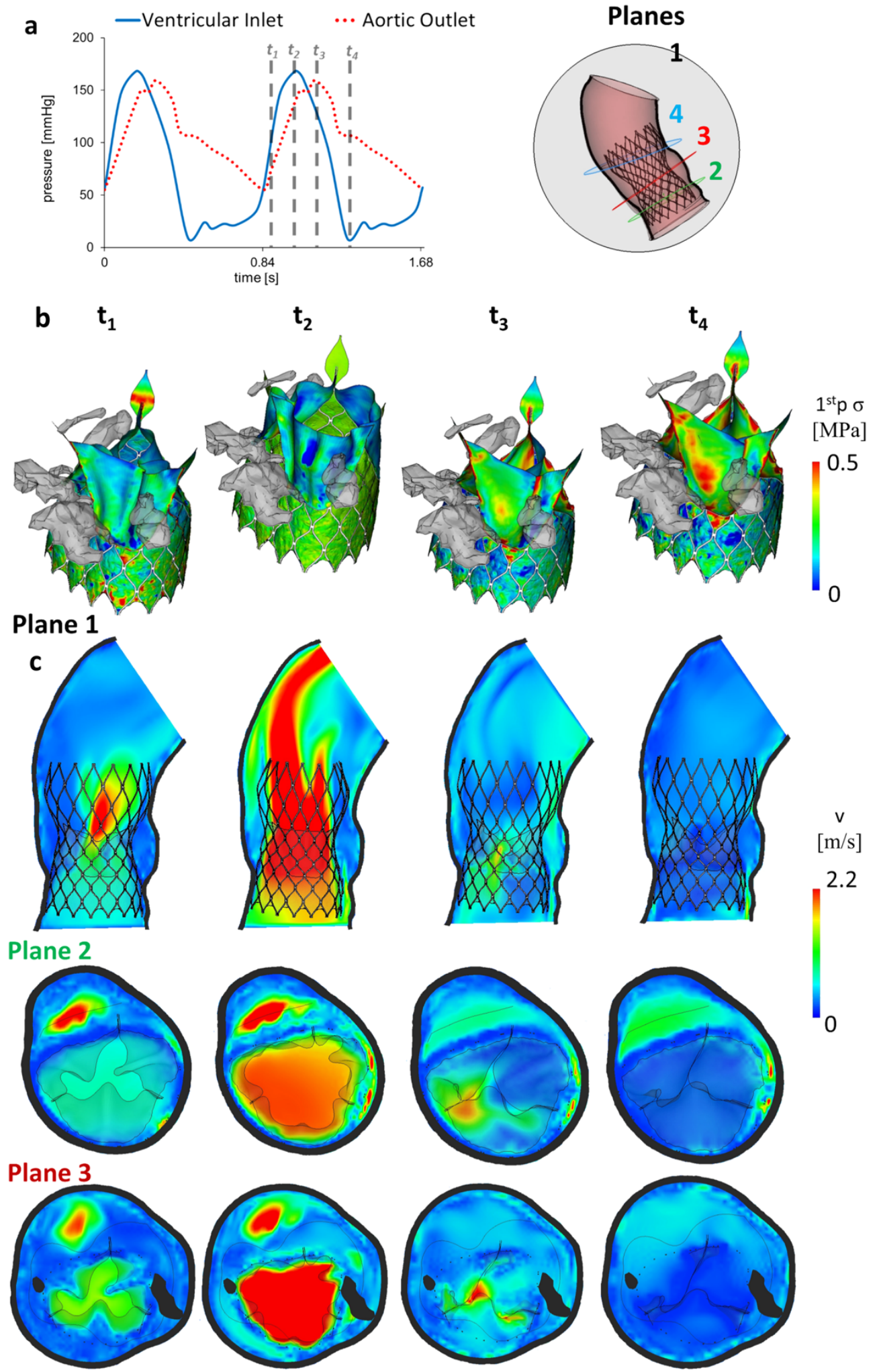

Plane 4
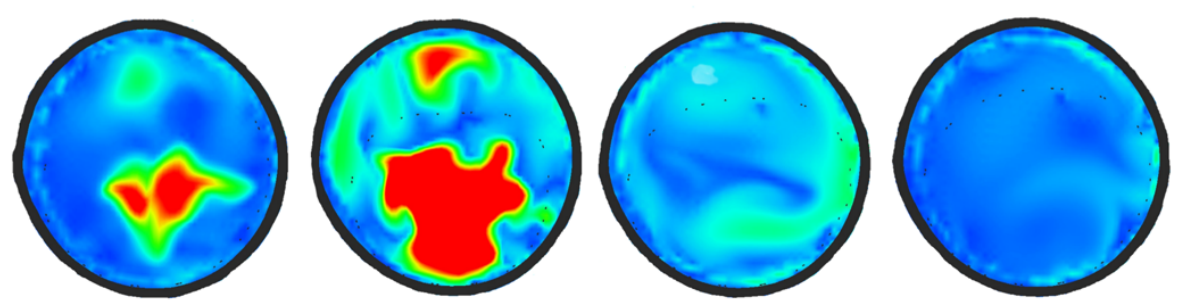

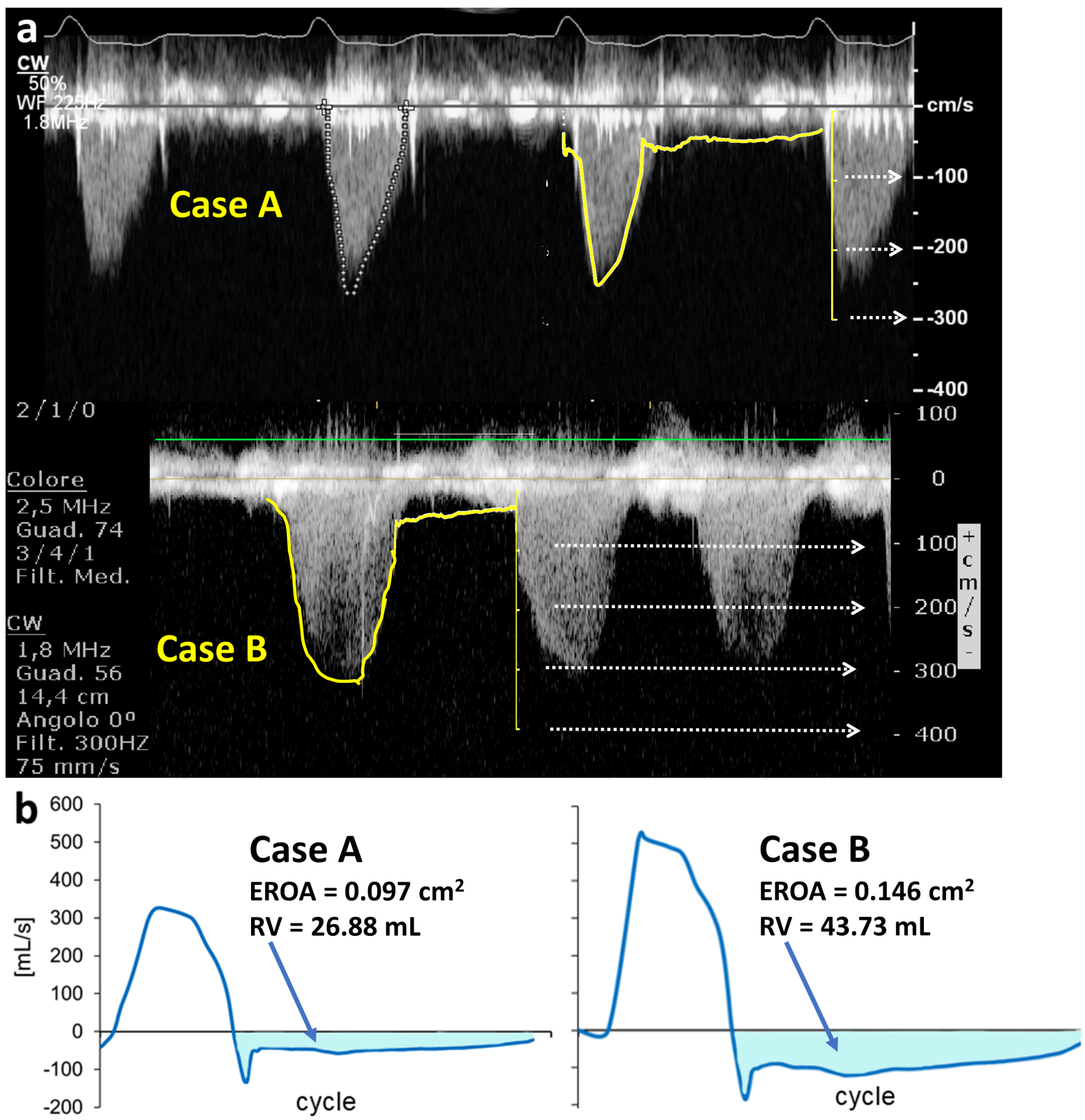

Figure 7 